\title{
The Global Asthma Network rationale and methods for Phase I global surveillance: prevalence, severity, management and risk factors
}

\author{
Philippa Ellwood (1) ${ }^{1}$, M. Innes Asher ${ }^{1}$, Nils E. Billo², Karen Bissell ${ }^{3,4}$, \\ Chen-Yuan Chiang ${ }^{4,5,6}$, Eamon M. Ellwood ${ }^{1}$, Asma El-Sony ${ }^{7}$, \\ Luis García-Marcos ${ }^{8}$, Javier Mallol ${ }^{9}$, Guy B. Marks ${ }^{10}$, Neil E. Pearce ${ }^{11}$ and \\ David P. Strachan ${ }^{12}$
}

\begin{abstract}
Affiliations: ${ }^{1}$ Paediatrics: Child and Youth Health, The University of Auckland, Auckland, New Zealand. ${ }^{2}$ Joensuu, Finland. ${ }^{3}$ International Union Against Tuberculosis and Lung Disease (The Union), Paris, France. ${ }^{4}$ School of Population Health, The University of Auckland, Auckland, New Zealand. ${ }^{5}$ Division of Pulmonary Medicine, Dept of Internal Medicine, Wan Fang Hospital, Taipei Medical University, Taipei, Taiwan. ${ }^{6}$ Internal Medicine, School of Medicine, College of Medicine, Taipei Medical University, Taipei, Taiwan. ${ }^{7}$ Epidemiological Laboratory (Epi-Lab) for Public Health, Research and Development, Khartoum, Sudan. ${ }^{8}$ Paediatric Allergy and Pulmonology Units, Arrixaca University Children's Hospital, University of Murcia and IMIB Bioresearch Institute, Murcia, Spain. ${ }^{9}$ Paediatric Respiratory Medicine, Hospital-CRS El Pino, University of Santiago de Chile, (USACH), Santiago, Chile. ${ }^{10}$ Dept of Medical Statistics, Respiratory and Environmental Epidemiology, South Western Sydney Clinical School, University of New South Wales, Sydney, Australia. ${ }^{11}$ Faculty of Epidemiology and Population Health, London School of Hygiene and Tropical Medicine, London, UK. ${ }^{12}$ Population Health Research Institute, St George's, University of London, London, UK.
\end{abstract}

Correspondence: Philippa Ellwood, The University of Auckland, Paediatrics: Child and Youth Health, Private Bag 92019, Auckland 1301, New Zealand. E-mail: p.ellwooddauckland.ac.nz

@ERSpublications

The Global Asthma Network undertakes global studies of asthma surveillance, management and risk factors http://ow.ly/h67s305t9vS

Cite this article as: Ellwood P, Asher MI, Billo NE, et al. The Global Asthma Network rationale and methods for Phase I global surveillance: prevalence, severity, management and risk factors. Eur Respir J 2017; 49: 1601605 [https://doi.org/10.1183/13993003.01605-2016].

ABSTRACT The Global Asthma Network (GAN), established in 2012, followed the International Study of Asthma and Allergies in Childhood (ISAAC). ISAAC Phase One involved over 700000 adolescents and children from 156 centres in 56 countries; it found marked worldwide variation in symptom prevalence of asthma, rhinitis and eczema that was not explained by the current understanding of these diseases; ISAAC Phase Three involved over 1187496 adolescents and children (237 centres in 98 countries). It found that asthma symptom prevalence was increasing in many locations especially in low- and middle-income countries where severity was also high, and identified several environmental factors that required further investigation.

GAN Phase I, described in this article, builds on the ISAAC findings by collecting further information on asthma, rhinitis and eczema prevalence, severity, diagnoses, asthma emergency room visits, hospital admissions, management and use of asthma essential medicines. The subjects will be the same age groups as ISAAC, and their parents. In this first global monitoring of asthma in children and adults since 2003, further evidence will be obtained to understand asthma, management practices and risk factors, leading to further recognition that asthma is an important non-communicable disease and to reduce its global burden. 


\section{Introduction}

An estimated 241 million people in all parts of the world suffer from asthma [1], and nearly 1000 people per day die from asthma [2]. The Global Asthma Network (GAN) [3] was established in 2012 to improve asthma care globally, with a focus on low- and middle-income countries, through enhanced surveillance, research collaboration, capacity building and access to quality-assured, affordable, essential asthma medicines. GAN was formed by scientists from the International Study of Asthma and Allergies in childhood (ISAAC) 1991-2012 (Phases One [4], Two [5] and Three [6]) and from the International Union Against Tuberculosis and Lung Disease (The Union) following production of the first Global Asthma Report (GAR) 2011 [7], launched in New York (NY, USA) in 2011 at the time of the United Nations High-Level meeting on non-communicable diseases. A second GAR [8], written and produced by GAN in 2014 was launched in October at the 45th Union World Conference on Lung Health, Barcelona, Spain. These reports highlight the existing evidence of the burden of asthma, provide evidence-based recommendations for cost-effective health improvement, especially in low- and middle-income countries and advocate for increasing the priority given to interventions for asthma.

ISAAC Phase One (1994-1995) identified large variations in the prevalence of symptoms of asthma, rhinitis and eczema, with differences of between 20 -fold and 60-fold between centres, showing marked differences in asthma prevalence in populations with similar genetic or ethnic backgrounds [9], suggesting that environmental risk factors in the broadest sense were the major determinants of the prevalence of asthma in a community. ISAAC Phase Three (2001-2003) examined time trends and included new questions on risk factors which identified many environmental associations [10]. These were the last ISAAC studies.

The Union implemented asthma pilot projects in Benin [11], China (Huaiyuan County) [12], El Salvador [13] and Sudan [14]. These projects found that inhaled corticosteroids were not available in any of these sites in the public sector and that asthma was greatly underdiagnosed. The Union's Asthma Drug Facility enabled countries to purchase quality-assured essential affordable asthma medicines; and inhaled corticosteroids became widely used, resulting in a reduction in hospitalisations $[15,16]$.

GAN Phase I is a development from ISAAC (particularly Phases One [17-21] and Three [22-24]), and The Union [11, 12, 14, 25]. GAN Phase I has extended its surveillance to include adults, for whom there are limited global data, and to assess asthma management, which is commonly suboptimal in settings with limited access to quality-assured essential asthma medicines.

GAN aims to progress recommendations in the GARs and produce a further report in 2018, engaging and reinforcing the role of Government's health ministers, the World Health Organization (WHO), policy-makers, health-workers, those with asthma, development partners/donors and the media, in efforts to improve asthma care globally. GANs core activities are global surveillance of asthma prevalence, severity, management and risk factors; promotion of standard case management of asthma; operational research; capacity building; engagement with policy makers; and access to affordable quality-assured essential medicines.

The four main hypotheses of GAN Phase I are: 1) globally, the burden of asthma is changing in adults and children; 2) there is large variation in the diagnosis of asthma; 3 ) in many locations, asthma is under-diagnosed and its management is suboptimal; and 4) there are potentially remedial risk factors of asthma.

\section{Methodology}

Study subjects

Each GAN centre is a defined geographical area. Within each centre, subjects will be selected in the same manner as ISAAC Phase Three. The same age groups will be used: 13/14-year-old adolescents (self-completed questionnaires) and 6/7-year-old children (parental completed questionnaires), and GAN Phase I adds their parents as an adult group (see next paragraph). The sampling unit will be a school for each age group which will be selected using a table of random numbers. A minimum of 10 schools will be required in each centre, or all schools in the sampling frame if there are fewer than 10 schools. Students of both age groups are selected either by grade/level/year or by age group.

Additionally, GAN is recommending that the parents/guardians of the adolescents and children are also surveyed in order to obtain symptom prevalence of asthma, rhinitis and eczema as there has been a paucity of international prevalence data on adult asthma. The GAR 2011 and GAR 2014 reported data on asthma in adults mainly from the World Health Survey 2003, which had small numbers of participants in

Support statement: The GAN Global Centre (Auckland, New Zealand) received funding from The Union (France) from 2012 to 2015. Funding information for this article has been deposited with the Open Funder Registry.

Conflict of interest: None declared. 
most countries and which was a general health survey with very limited information specifically on asthma and wheeze. In addition the adults in GAN will provide more in-depth environmental data, particularly from the parents of the adolescent group, as the adolescents may not be able to answer some questions relating to their early life.

\section{Study design}

GAN Phase I is a cross-sectional, multi-centre, multi-country, epidemiological research, study design. This will meet these objectives of GAN Phase I: 1) to conduct asthma surveillance around the world in two age groups of children, and their parents, measuring prevalence, severity, management and risk factors, using similar methods to ISAAC Phase Three; 2) to examine time trends in prevalence, severity and risk factors from centres participating in ISAAC Phase Three; and 3) to evaluate the appropriateness of asthma management, especially access to quality-assured essential asthma medicines, as defined by WHO.

\section{Methods}

The methodology will be the same as ISAAC Phase Three methodology, with expansion, in particular, in the areas of on risk factor determinants and on management of asthma. The manual containing the information required to undertake the fieldwork is available from the GAN website [3]. Each centre has a defined geographical area and the centres that undertook ISAAC Phase Three and/or Phase One are expected to use the same sampling frame so that comparisons of data can be subsequently undertaken. As in ISAAC, new centres are encouraged to join to obtain wider diverse global participation and to encourage rural/urban comparisons. Principal investigators are identified at the registration stage which began in November 2015. Phase I will be co-ordinated by N. Pearce (London School of Hygiene and Tropical Medicine, London, UK) who will lead the main data centre. L. García-Marcos (IMIB Bioresearch Institute and University of Murcia, Murcia, Spain) will lead the data centre for Spanish and Portuguese language centres. The GAN Global Centre, will be led by I. Asher (the University of Auckland, Auckland, New Zealand) assisted by P. Ellwood, GAN Research Manager.

An Expression of Interest form was available from 2012, from the GAN website until formal registrations opened on November 1, 2015. As of July 1, 2016, there were expressions of interest from 344 centres in 132 countries, with formal registrations from 89 centres in 35 countries. The registration document identifies principal investigators (two may be appointed per centre, one for adolescents and children and the other for the adult group), and a national coordinator may be appointed if there is more than one centre in a country and if there is an identified need. The manual contains all the necessary information needed to undertake the fieldwork and describes the criteria required for inclusion in the worldwide data set.

The same standardised written core questionnaires, developed for ISAAC for use in Phases One and Three, will be used in GAN. Demographic questions include the participant's name, where allowed, age, date of birth, school (for the adolescents and children), sex and date of interview. Questionnaires will be coded using a unique number for each centre, school and participant to ensure confidentiality and to link the questionnaires between the adults, adolecents and children. Collection of data regarding participants ethnicity is optional in which case ethnic categories from local census forms should be used [26].

The written core questionnaires, used in ISAAC, have had a question about doctor-diagnosed asthma, rhinitis and eczema added. The core questions are both sensitive and specific, have good content, construct and concurrent and predictive validity [27]. Height and weight measurements will be taken by the fieldworkers in schools. The environmental risk factor questionnaire, developed for ISAAC Phase Three has been expanded for use in GAN. Not all of these questions are compulsory as indicated in the GAN Manual. Some questions that were not considered appropriate for the adolescent group to complete in ISAAC Phase Three have been included in the adult questionnaire. The adult symptoms questionnaire was derived from elements from ISAAC and the European Community Respiratory Health Survey with the aim of capturing the spectrum of chest symptoms that might be attributable to asthma in young and middle-aged adults and the diagnostic labels applied (see the supplementary material).

As in ISAAC, a video asthma questionnaire will be an optional tool: the international version as used in ISAAC [28]. This 6-min non-verbal video shows clinical signs of asthma symptoms and was developed by the Wellington Asthma Research Group, to avoid problems of translation and comprehension of terms such as "wheeze" or "whistling" and their use in culturally heterogeneous populations [29]. The video has the advantage of obtaining data from a large number of students quickly and efficiently.

\section{Sample size and subjects}

As in ISAAC, a sample size of 3000 participants per age group (and therefore potentially 6000 adults of each group) will be used. The sample size will provide greater than $99 \%$ (at the $1 \%$ level of significance) to detect differences in the prevalence of wheezing of $30 \%$ in one centre and $25 \%$ in another centre [27]. As 
sampling is done by school, while the information is gained from the school pupils and adults, there is likely to be a cluster effect. Like ISAAC, the analysis will incorporate adjustment for cluster sampling using the design effect [30], which is important for large studies where clusters of different size may be used in different regions. If centres are unable to obtain 3000 participants, provided they do not have fewer than 1000 per age group and fulfil the criteria described in the GAN manual, they may be included.

The mean participation in ISAAC Phase Three was $88 \%$ in adolescents and $84.5 \%$ in children. High participation is sought for GAN Phase I: at least $80 \%$ for $13 / 14$ year olds and $70 \%$ for $6 / 7$ year olds and $70 \%$ for adults/parents. For the adolescent group, a return visit to school can be made to capture the absentees and, for the children, redistribution of questionnaires to the parents can be done once or, if necessary, twice.

\section{Analyses}

On-line documents for data entry are available from the GAN website. Once the centres have registered, principal investigators are emailed a personalised copy of the centre report for use during fieldwork. The centre report can be completed on-line and submitted via the website to the GAN Global centre in Auckland, New Zealand.

At the time of conducting the survey, fieldworkers are requested to check the questionnaires for any obvious demographic entry errors so that this information can be corrected using school records. No alteration to the symptom and risk factor questionnaire data is allowed. Data is entered onto the computer as it is presented on the questionnaire, preserving confidentiality by coding. At least $10 \%$ of the data must be double entered to identify data entry errors. Centre principal investigators are responsible for their own data coding and entry. An epi info package is available on the GAN website for investigators to use. Centres may wish to use questionnaire scanning software such as optical mark recognition for data entry; however, procedures to deal with data entry errors must be documented and sent to the GAN Global Centre. The software should have the ability to export the data set as a .CSV file.

Data will be submitted to the GAN Global Centre (Auckland, New Zealand) at the same time the centre report is submitted. The GAN Global Centre will acknowledge receipt of the data and centre report, will undertake some quality control checks and will then send the data to one of two designated data centres. The data centre in Murcia, led by L. García-Marcos will undertake the data checks on all Spanish- and Portuguese-speaking centres. The data centre in London, led by N. Pearce will undertake the data checks for centres using all other languages.

The approach to data analyses will be similar to that used in ISAAC [6]. This standardised approach is particularly important for examination of time trends in prevalence and severity from ISAAC to GAN [31, 32], to ensure comparability. For the environmental questions, the approach used will follow that of ISAAC [33], and assessment of time trends in risk factors will allow changes in asthma prevalence to be analysed in relation to changes in prevalence of established risk factors within the centres which participated in ISAAC Phase Three.

On completion of the data checks and methodology checks from the centre report, any deviations from protocol will be examined carefully by the GAN Steering Group. Provided the deviation from protocol is not severe enough for exclusion of the centre, the data will be included in the analyses and subsequent publications. Protocol variations that are accepted will be footnoted in the tables of the publications. This follows the same principles as adopted in ISAAC Phases One and Three.

\section{Ownership of data, ethics, dissemination of results and funding}

Each centre owns its own data and is free to publish these data without prior approval of the GAN Steering Group. All worldwide publications will have a writing group "and the Global Asthma Network Phase I Study Group”. This group, comprising all Steering Group members, national coordinators (if one has been appointed) and principal investigators of centres included in the paper, will be named in each paper and consulted on the paper in preparation prior to journal submission. Each centre is required to obtain ethics approval from their local ethics committee prior to the start of their study. Each centre is required to provide funding for the fieldwork.

\section{Piloting of GAN Phase I}

The pilot of the GAN methodology and questionnaires for the adolescent group, the children and the parents/adults of both groups was undertaken in one school for each age group in Auckland, New Zealand, Cartagena, Spain, and Madani, in east-central Sudan. The adolescents completed the questionnaire at school and gave immediate feedback which enabled some question formats to be improved. The questions were discussed with the students, for example, if they found the questions on smoking intrusive. Most participants were appreciative of the anonymity of questionnaires, although some 
found it a bit long. For the children and for the parent/adults of both groups, the questionnaires were sent home for completion and return (for the parent/adults, this was a new methodology). Investigators are encouraged to include the parent/adult group, as the information provided will give new information on adults, and additional useful family history.

\section{Significance of GAN}

GAN will expand on the ISAAC Phase Three time trends findings to explore in depth the negative and positive associations found with the environmental risk factor data. In addition, this will be the first major global surveillance of asthma prevalence, severity, management and risk factors which includes adults/ parents of two age groups of school children. GAN will also collect further information on the topics addressed in the Global Asthma Reports 2011 and 2014: including asthma prevalence and severity; diagnosis of asthma; unplanned visits including emergency room visits and hospital admissions; management policies; and access to quality-assured essential asthma medicines. New centres from countries that had not previously undertaken ISAAC will provide rich new information.

GAN Phase I will build on the findings of ISAAC by collecting further information on asthma, rhinitis and eczema prevalence, severity, diagnoses, asthma emergency room visits, hospital admissions, management and use of quality-assured essential asthma medicines. The subjects will be the same age groups as ISAAC Phase Three, and their parents. In this first global monitoring of asthma in children and adults since 2003, further evidence will be obtained to understand asthma, management practices and risk factors, leading to further recognition of asthma as an important non-communicable disease and to reduce its global burden.

\section{Acknowledgements}

The GAN Steering Group would like to acknowledge the ground-breaking work of the ISAAC Steering Committee in establishing and conducting worldwide asthma epidemiological studies of asthma rhinitis and eczema. They would like to thank the GAN Phase I Collaborators who will be named in the first worldwide publication. We also thank The Union (France) for providing essential core funding for the GAN Global Centre (Auckland, New Zealand) from 2012 to 2015.

\section{References}

1 Global Burden of Disease Study 2013 Collaborators. Global, regional, and national incidence, prevalence, and years lived with disability for 301 acute and chronic diseases and injuries in 188 countries, 1990-2013: a systematic analysis for the Global Burden of Disease Study 2013. Lancet 2015; 386: 743-800.

2 GBD 2013 Mortality and causes of Death Collaborators. Global, regional, and national age-sex specific all-cause and cause-specific mortality for 240 causes of death, 1990-2013: a systematic analysis for the Global Burden of Disease Study 2013. Lancet 2015; 385: 117-171.

3 Global Asthma Network Steering Group. The Global Asthma Network Website: The Global Asthma Network, 2012. www.globalasthmanetwork.org Date last accessed: July 2016; Date last updated: June 2016.

4 Asher MI, Keil U, Anderson HR, et al. International Study of Asthma and Allergies in Childhood (ISAAC): rationale and methods. Eur Respir J 1995; 8: 483-491.

5 Weiland SK, Husing A, Strachan DP, et al. Climate and the prevalence of symptoms of asthma, allergic rhinitis, and atopic eczema in children. Occup Environ Med 2004; 61: 609-615.

6 Ellwood P, Asher MI, Beasley R, et al. The International study of Asthma and Allergies in Childhood (ISAAC): Phase Three rationale and methods. Int J Tuberc \& Lung Dis 2005; 9: 10-16.

7 The International Union Against Tuberculosis and Lung Disease. Paris. France, editor. The Global Asthma Report. www.globalasthmanetwork.org Date last accessed: July 2016; Date last updated: September 2011.

8 Global Asthma Network Steering Group, editor. The Global Asthma Report. www.globalasthmanetwork.org Date last accessed: July 2016; Date last updated: October 2014.

9 Beasley R, Ellwood P, Asher I. International patterns of the prevalence of pediatric asthma the ISAAC program. Pediatr Clin North Am 2003; 50: 539-553.

10 ISAAC Phase Three. http://isaac.auckland.ac.nz/phases/phasethree/phasethree.html Date last accessed: July 2016; Date last updated: November 2015. Website Publications

11 Gininafon M, Tawo L. Asthma Management: Country Profiles: Benin. www.globalasthmareport.org/2011/ management/country_profiles/Benin_Profile.pdf Date last accessed: July 2016; Date last updated: September 2011.

12 Kan XH, Chiang CY, Enarson DA, et al. Asthma as a hidden disease in rural China: opportunities and challenges of standard case management. Public Health Action 2012; 2: 87-91.

13 Castillo F, Garay J. Asthma Management: Country Profiles: El Salvador. www.globalasthmareport.org/2011/ management/country_profiles/ElSalvador_Profile.pdf Date last accessed: July 2016; Date last updated: September 2011.

14 El Sony A, Chiang CY, Malik E, et al. Standard case managment of asthma in Sudan: a pilot project. Public Health Action 2013; 3: 247-252.

15 El Sony A, Ait-khaled N, Mallol J. Asthma as a Lung Health Priority in Low- and Middle-Income Countries, Chapter 12. www.globalasthmareport.org/resources/Global_Asthma_Report_2014.pdf Date last accessed: July 2016; Date last updated: October 2014.

16 Bissell K, Chiang CY, Ait-Khaled N, et al. Asthma Management in Low-Income Countries. www. globalasthmareport.org/resources/Global_Asthma_Report_2014.pdf Date last accessed: July 2016; Date last updated: October 2014.

17 ISAAC Steering Committee. Worldwide variation in prevalence of symptoms of asthma, allergic rhinoconjunctivitis, and atopic eczema: ISAAC. The International Study of Asthma and Allergies in Childhood (ISAAC) Steering Committee. Lancet 1998; 351: 1225-1232. 
18 ISAAC Steering Committee. Worldwide variations in the prevalence of asthma symptoms: the International Study of Asthma and Allergies in Childhood (ISAAC). Eur Respir J 1998; 12: 315-335.

19 Strachan D, Sibbald B, Weiland S, et al. Worldwide variations in prevalence of symptoms of allergic rhinoconjunctivitis in children: the International Study of Asthma and Allergies in Childhood (ISAAC). Pediatr Allergy Immunol 1997; 8: 161-176.

20 Williams H, Robertson C, Stewart A, et al. Worldwide variations in the prevalence of symptoms of atopic eczema in the International Study of Asthma and Allergies in Childhood. J Allergy Clin Immunol 1999; 103(): 125-138.

21 Mallol J, Crane J, von Mutius E, et al. The International Study of Asthma and Allergies in Childhood (ISAAC) Phase Three: A global synthesis. Allergol Immunopathol (Madr) 2013; 41: 73-85.

22 Lai K, Beasley R, Crane J, et al. Global variation in the prevalence and severity of asthma symptoms: Phase Three of the International study of Asthma and allergies in Childhood (ISAAC). Thorax 2009; 64: 476-483.

23 Ait-Khaled N, Pearce N, Anderson H, et al. Global map of the prevalence of symptoms of rhinoconjunctivitis in children: The International Study of Asthma and Allergies in Childhood (ISAAC) Phase Three. Allergy 2009; 64: 123-148.

24 Odhiambo J, Williams $\mathrm{H}$, Clayton TO, et al. Global variations in prevalence of eczema symptoms in children from ISAAC Phase Three. J Allergy Clin Immunol 2009; 124: 1251-1258.

25 Ade G, Gninafon M, Tawo L, et al. Management of asthma in Benin: the challenge of loss to follow-up. Public Health Action 2013; 3: 76-80.

26 Ellwood P, Asher I, Ellwood E, et al. Global Asthma Network Phase I Manual. Global Surveillance: Prevalence, Severity, Management and Risk Factors. Auckland, New Zealand, 2015. www.globalasthmanetwork.org/ surveillance/manual/manual.php Date last accessed: July 2016; Date last updated: August 2015.

27 ISAAC Steering Committee. International Study of Asthma and Allergies in Childhood. 2nd Edn. Auckland/ Münster, ISAAC Phase One Manual, 1993.

28 Wellington Asthma Research Group. ISAAC International Video. Wellington, Wellington Asthma Research Group, 1995. www.globalasthmanetwork.org/surveillance/manual/video.php Date last accessed: July 2016; Date last updated: February 1995.

29 Crane J, Mallol J, Beasley R, et al. Agreement between written and video questions for comparing asthma symptoms in ISAAC. Eur Respir J 2003; 21: 455-461.

30 Rao JN, Scott AJ. A simple method for the analysis of clustered binary data. Biometrics 1992; 48: 577-585.

31 Lai CKW, Beasley R, Crane J, et al. Global variation in the prevalence and severity of asthma symptoms: Phase Three of the International Study of Asthma and Allergies in Childhood (ISAAC). Thorax 2009; 64: 476-483.

32 Pearce N, Aït-Khaled N, Beasley R, et al. Worldwide trends in the prevalence of asthma symptoms: Phase III of the International Study of Asthma and Allergies in Childhood (ISAAC). Thorax 2007; 62: 758-766.

33 Ellwood P, Asher MI, Garcia-Marcos L, et al. Do fast foods cause asthma, rhinoconjunctivitis and eczema? Global findings from the International Study of Asthma and Allergies in Childhood (ISAAC) Phase Three. Thorax 2013; 68: $351-360$ 